\title{
A INFLUÊNCIA DO ANEL NA EVOLUÇÃO PONDERAL APÓS QUATRO ANOS DA DERIVAÇÃO GÁSTRICA EM Y-DE-ROUX LAPAROSCÓPICA
}

\author{
Ring influence on ponderal evolution after four years of laparoscopic Roux-en-Y gastric bypass \\ Irineu RASERA-JUNIOR ${ }^{1,3}$, Natalia Moreno GAINO$^{1}$, Maria Rita Marques de OLIVEIRA $^{2}$, Patrícia Fátima Sousa \\ NOVAIS ${ }^{1,4}$, Celso Vieira de Souza LEITE $^{3}$, Maria Aparecida Coelho de Arruda HENRI ${ }^{3}$
}

Trabalho realizado na ${ }^{1}$ Clínica Bariátrica, Hospital Fornecedores de Cana, Piracicaba, SP; 2 Instituto de Biociências da Universidade Estadual Paulista (UNESP), Botucatu, SP; ${ }^{3}$ Faculdade de Medicina da Universidade Estadual Paulista (UNESP), Botucatu, SP; ${ }^{4}$ Faculdade de Ciências Farmacêuticas da Universidade Estadual Paulista (UNESP), Araraquara, SP, Brasil.

DESCRTORES - Derivação gástrica. Gastroplastia. Cirurgia bariátrica. Perda de peso.
RESUMO - Racional - A utilização de anel nas derivações gástricas em Y-de-Roux ainda é motivo de polêmica entre os cirurgiões bariátricos. Não há consenso quanto às suas repercussões em relação à perda ponderal e à manutenção do peso em longo prazo. Objetivo - Avaliar a influência do anel sobre a evolução do peso corporal no decorrer de quatro anos após operação bariátrica. Método - Foram analisadas retrospectivamente 143 mulheres submetidas à derivação gástrica em Y-de-Roux videolaparoscópica pareadas pela utilização ou não do anel de Silastic $®$. O tempo de seguimento foi de até 48 meses. Os critérios de inclusão foram idade superior a 18 anos, operação bariátrica primária e frequência regular à clínica no período de interesse para a pesquisa. A técnica manteve reservatório gástrico de pequena curvatura, volume estimado em 30 $\mathrm{ml}$. A alça alimentar media $150 \mathrm{~cm}$ e a biliar $40 \mathrm{~cm}$ a partir do ângulo duodenojejunal. O grupo "com anel" utilizou anel tubular de Silastic $®$ com comprimento de 6,5 cm, colocado à $2 \mathrm{~cm}$ da anastomose gastrojejunal. O anel era fechado por cinco nós com fio de polipropileno em seu interior. Na manhã seguinte ao procedimento cirúrgico as pacientes recebiam líquidos isotônicos; no segundo dia dieta líquida salgada sem resíduos e alta hospitalar no terceiro dia. Dieta pastosa iniciava a partir do 20 o dia e sólida no 30o, juntamente com uma drágea diária de polivitamínico. Resultados - O emagrecimento do grupo com anel foi maior que o sem anel em todos os períodos analisados a nível de $10 \%$ e de $5 \%$ apenas no 30 ano pós-operatório. A proporção das operadas que não atingiram perda do excesso de peso de $50 \%$ foi significativamente maior no grupo sem anel que no grupo com anel (31\% entre as sem anel e $8 \%$ das com anel no 40 ano). Não houve diferença entre os grupos na recuperação tardia do peso perdido na operação. Conclusões - Os resultados foram favoráveis à utilização do anel ao se analisar exclusivamente a perda de peso.

\section{Correspondência:}

Irineu Rasera Júnior,

e-mail: irineu@bariatrica.com.br

Fonte de financiamento: não há Conflito de interesses: não há

Recebido para publicação: 02/04/2012 Aceito para publicação: 03/08/2012

HEADINGS - Gastric bypass. Gastroplasty. Bariatric surgery. Weight loss.
ABSTRACT - Background - Use of ring in Roux-en-Y gastric bypass is still a matter of controversy among bariatric surgeons. There is no consensus on its impact in relation to weight loss and weight maintenance in the long term. Aim - To evaluate the influence of the ring on the evolution of body weight over four years after bariatric surgery. Methods - Retrospective analyzis of 143 women who underwent laparoscopic Roux-en-Y gastric bypass paired on the use or not use of Silastic ${ }^{\circledR}$ ring. Follow-up time was 48 months. Inclusion criteria were age over 18 years, primary bariatric operation and regular attendance at the clinic during the period of interest for research. The technique kept small gastric reservoir estimated in a volume of $30 \mathrm{ml}$. The food limb had in average $150 \mathrm{~cm}$ and the bile one $40 \mathrm{~cm}$ from the duodenojejunal

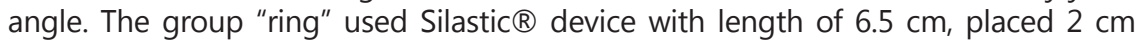
from gastrojejunal anastomosis. The ring was closed for five polypropylene surgical thread sutures. In the morning after surgery the patients received isotonic fluids; on the second day salty liquid diet and were discharged on the third day. Semisolid diet started from the 20th day and solid on the 30th, with daily tablet of polivitamins. Results - The weight loss was larger on the ring than without ring groups in all periods, respectively $10 \%$ and only $5 \%$ in the third postoperative year. The proportion of not having reached the $50 \%$ excess weight loss expectative was significantly higher in the group without ring than in the group with the ring (31\% and $8 \%$ respectively in the fourth year). There was no difference between groups in delayed recovery of weight lost with the operation. Conclusions - The results were favorable to use the ring exclusively when it is analyzed only the weight loss. 
INTRODUÇÃO

utilização de anel nas derivações gástricas
em Y-de-Roux (DGYR) ainda é motivo de
polêmica entre os cirurgiões bariátricos. Considerada o "padrão-ouro" das operações para controle do peso corporal ${ }^{5}$ em obesos mórbidos, a DGYR inclui o uso opcional de prótese na porção terminal da bolsa - o anel ou bandagem de contenção gástrica. Entretanto, ainda não há consenso quanto às repercussões causadas por esse aumento na restrição gástrica em relação à perda ponderal e à manutenção do peso em longo prazo.

O uso de constritores da bolsa gástrica teve início com Linner \& Drew em 19859,17, que empregaram um elemento de contenção para prevenir possível dilatação da gastroenterostomia. Posteriormente, Fobi $^{11}$ e Capella ${ }^{6}$ propuseram modificações nas DGYR que incluíam materiais sintéticos no extremo distal da bolsa gástrica para restringir o esvaziamento.

A colocação do anel a montante da anastomose gastrojejunal é imputada como alternativa eficaz e duradoura para potencializar a perda de peso em pacientes obesos mórbidos, embora os resultados intermediários e tardios ainda sejam relativamente escassos na literatura ${ }^{21,22}$.

Após as operações bariátricas a redução do peso é claramente visível, com consequente melhora das comorbidezes e da qualidade de vida. No entanto, o indivíduo pode recuperar parcialmente ou até totalmente o peso perdido se não adquirir mudanças efetivas no estilo de vida relativas às práticas dietéticas e de atividades físicas ${ }^{12}$. Alguns estudos apontaram uma incômoda recuperação ponderal no pós-operatório tardio ${ }^{7,18}$, principalmente entre $\circ 3^{\circ}$ e $\circ 5^{\circ}$ ano pósoperatório. A não utilização do anel de contenção pode estar relacionada a um maior aumento tardio de peso em comparação aos pacientes que não têm essa

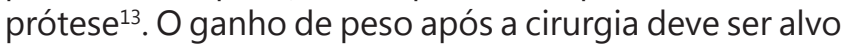
de estudo e monitoramento, com vistas à preservação dos benefícios obtidos.

Considerando que são poucos os trabalhos avaliando se o fator restritivo adicional imposto por esse anel ou bandagem interfere significativamente na perda e manutenção do peso, o presente estudo se justifica. O objetivo foi avaliar a influência do anel sobre a evolução do peso corporal no decorrer de quatro anos após cirurgia bariátrica.

\section{MÉTODO}

O estudo envolveu 143 mulheres operadas na Clínica Bariátrica - Hospital dos Fornecedores de Cana de Piracicaba - São Paulo, acreditada pela Surgical Review CorporationTM. Essas mulheres foram agrupadas quanto à presença (com anel: $n=75)$ ou ausência (sem anel: $n=68$ ) do anel de restrição e ao tempo de seguimento $(12,24,36$ e 48 meses). Trata-se de um estudo retrospectivo dos registros em prontuário no período de 1999 a 2005. Para a inclusão na pesquisa foram utilizados os seguintes critérios: idade superior a 18 anos, cirurgia bariátrica primária e frequência regular à clínica no período de interesse para a pesquisa. Foram incluídas na pesquisa as mulheres que concordaram em participar do estudo, após terem assinado o termo de consentimento livre e esclarecido concordando com o estudo retrospectivo. O estudo foi aprovado pelo comitê de Ética em Pesquisa da Universidade Estadual Paulista da Faculdade de Ciências Farmacêuticas de Araraquara sob o número 16/2006. Na tabela 1 pode ser verificado que o peso e a idade das participantes do estudo foram semelhantes em ambos os grupos.

A técnica utilizada foi a DGYR videolaparoscópica, com reservatório gástrico de pequena curvatura, volume estimado em $30 \mathrm{ml}$ e anastomose gastrojejunal manual em dois planos com dimensões entre 1,5 e $2,0 \mathrm{~cm}$. A alça alimentar mede $150 \mathrm{~cm}$ e a biliar $40 \mathrm{~cm}$ a partir do ângulo de Treitz. O tempo médio de cirurgia foi de 130 minutos, variando de 250 a 90 minutos, das primeiras em relação às mais recentes. A drenagem da cavidade com dreno túbulo-laminar a vácuo foi rotineira. $O$ grupo "com anel" utilizou anel tubular de Silastic $®$, com comprimento de $6,5 \mathrm{~cm}$, colocado a uma distância de dois $\mathrm{cm}$ da anastomose gastrojejunal. $\mathrm{O}$ anel é fechado por cinco nós com fio de polipropileno em seu interior.

TABELA 1 - Idade e peso pré-operatório conforme o tipo de operação e o tempo de seguimento

\begin{tabular}{|c|c|c|c|c|c|c|c|c|c|c|c|}
\hline & \multicolumn{5}{|c|}{ Sem anel } & \multicolumn{5}{|c|}{ Com anel } & \multirow[b]{2}{*}{$p^{*}$} \\
\hline & & $\mathrm{n}$ & P25 & P50 & P75 & & $n$ & P25 & P50 & P75 & \\
\hline \multirow{4}{*}{$\begin{array}{l}\text { Idade } \\
\text { (anos) }\end{array}$} & $12 \mathrm{~m}$ & 68 & 29 & 34 & 42 & $12 \mathrm{~m}$ & 75 & 30 & 38 & 43 & 0,167 \\
\hline & $24 \mathrm{~m}$ & 61 & 29 & 34 & 42 & $24 \mathrm{~m}$ & 75 & 30 & 38 & 43 & 0,122 \\
\hline & $36 \mathrm{~m}$ & 56 & 29 & 34 & 42 & $36 \mathrm{~m}$ & 75 & 30 & 38 & 44 & 0,081 \\
\hline & $48 \mathrm{~m}$ & 52 & 29 & 34 & 41 & $48 \mathrm{~m}$ & 49 & 29 & 40 & 45 & 0,141 \\
\hline \multirow{4}{*}{ Excesso de peso (\%) } & $12 \mathrm{~m}$ & 68 & 74 & 88 & 101 & $12 \mathrm{~m}$ & 75 & 76 & 84 & 102 & 0,891 \\
\hline & $24 \mathrm{~m}$ & 61 & 76 & 90 & 101 & $24 \mathrm{~m}$ & 75 & 76 & 84 & 102 & 0,577 \\
\hline & $36 \mathrm{~m}$ & 56 & 77 & 89 & 101 & $36 \mathrm{~m}$ & 75 & 75 & 83 & 104 & 0,638 \\
\hline & $48 \mathrm{~m}$ & 52 & 76 & 88 & 100 & $48 \mathrm{~m}$ & 49 & 76 & 81 & 105 & 0,968 \\
\hline \multirow{4}{*}{ Peso (kg) } & $12 \mathrm{~m}$ & 68 & 103 & 113 & 125 & $12 \mathrm{~m}$ & 75 & 104 & 112 & 125 & 0,932 \\
\hline & $24 \mathrm{~m}$ & 61 & 105 & 114 & 125 & $24 \mathrm{~m}$ & 75 & 104 & 112 & 125 & 0,619 \\
\hline & $36 \mathrm{~m}$ & 56 & 105 & 113 & 125 & $36 \mathrm{~m}$ & 75 & 104 & 112 & 124 & 0,800 \\
\hline & $48 \mathrm{~m}$ & 52 & 104 & 113 & 125 & $48 \mathrm{~m}$ & 49 & 105 & 113 & 127 & 0,750 \\
\hline
\end{tabular}

P75 = terceiro quartil (75\%). $\mathrm{m}$ = meses após o procedimento cirúrgico. O excesso de peso foi calculado a partir da Metropolitan Height and Weight tables, 1983 
$\mathrm{Na}$ manhã seguinte ao procedimento cirúrgico as pacientes recebiam água, água-de-coco e isotônicos e, no segundo dia do pós-operatório, dieta líquida salgada sem resíduos. Alta hospitalar no terceiro dia pósoperatório. Dieta pastosa se iniciava a partir do vigésimo dia do pós-operatório e a sólida no trigésimo dia, juntamente com uma drágea diária de polivitamínico. Os históricos foram obtidos nos prontuários eletrônicos no sistema informatizado do centro de cirurgia, coletandose dados referentes ao peso corporal no pré-operatório e a cada 12 meses após a cirurgia.

Com as mulheres agrupadas conforme o tipo de cirurgia e o tempo de seguimento, foram analisados a perda do excesso de peso e a recuperação do excesso de peso em relação ao menor peso alcançado após a cirurgia. O valor do excesso de peso foi obtido a partir da diferença entre o peso pré-operatório e o peso ideal, segundo padrões internacionais ${ }^{19}$. As mulheres que permaneceram no estudo até os 48 meses foram avaliadas de maneira pareada.

\section{Análise dos dados}

Os dados foram tabulados, com os valores absolutos de peso transformados em valores relativos e expressos em mediana das percentagens. As comparações entre as medianas foram realizadas pelo teste de Mann-Whitney, após constatação das características não paramétricas dos dados. As comparações entre mais de dois grupos de dados foram feitas pelo teste de Friedman. As proporções entre variáveis categóricas dicotômicas foram testadas pelo teste Exato de Fisher. O nível de significância adotado foi de $5 \%$. As análises foram realizadas com auxílio do programa de computador BioEstat $3 \circledR$.

\section{RESULTADOS}

O emagrecimento do grupo "com anel" pode ser considerado maior no grupo "sem anel" em todos os períodos analisados $(12 \mathrm{~m}=74 \% \times 72 \% ; 24 \mathrm{~m}=79 \% \times$ $76 \% ; 36 \mathrm{~m}=76 \%$ × $72 \%$ e $48 \mathrm{~m}=76 \% \times 71 \%$ ) se adotado o nível de significância de $10 \%$ e foi significante no terceiro ano pós-operatório no nível de $5 \%$ (tabela 2).

Ao se avaliar o número de pacientes cuja perda do excesso de peso foi inferior a $50 \%$, verificou-se que a proporção dos que não atingem esse emagrecimento em cada período é significativamente maior no grupo "sem anel" que no grupo "com anel" até 48meses ( $12 \mathrm{~m}$ $=25,9 \% \times 2,7 \% ; 24 \mathrm{~m}=23,4 \% \times 2,7 \% ; 36 \mathrm{~m}=28,8 \% \times$ $5,3 \%$ e $48 \mathrm{~m}=30,9 \% \times 8,2 \%$ ) (tabela 3$)$.
TABELA 3 - Frequência de perda do excesso de peso inferior a $50 \%$ nos grupos "sem anel" e "com anel"

\begin{tabular}{|c|c|c|c|c|c|}
\hline & \multicolumn{2}{|c|}{$\begin{array}{c}\text { Sem anel - Perda de } \\
\text { peso }<50 \% \text { do excesso }\end{array}$} & \multicolumn{2}{|c|}{$\begin{array}{c}\text { Com anel - Perda de } \\
\text { peso }<50 \% \text { do excesso }\end{array}$} & \multirow[t]{2}{*}{$p^{*}$} \\
\hline & $n$ & $\%$ & $n$ & $\%$ & \\
\hline 12 meses & 14 & 25,9 & 2 & 2,7 & 0,001 \\
\hline 24 meses & 15 & 23,4 & 2 & 2,7 & 0,001 \\
\hline 36 meses & 17 & 28,8 & 4 & 5,3 & 0,001 \\
\hline 48 meses & 17 & 30,9 & 4 & 8,2 & 0,006 \\
\hline
\end{tabular}

*Teste exato de Fisher

Na figura 1 é apresentada a análise pareada das mulheres dos grupos "com anel" e "sem anel" acompanhadas por 4 anos. Verifica-se que ambos os grupos apresentaram perda de excesso de peso $>50 \%$ mantida até o quarto ano pós-cirúrgico. A curva de perda do excesso de peso de ambos os procedimentos cirúrgicos mostra-se acentuada no primeiro ano pósoperatório, com estabilização após o segundo ano. As curvas mantêm distanciamento constante entre si. Nesse sentido, mesmo que sendo excluídas dos grupos as mulheres seguidas por menos de 48 meses, as diferenças encontradas no percentual de perda de excesso de peso entre os grupos "com anel" e "sem anel" no segundo e terceiro ano pós-operatórios foram mantidas.

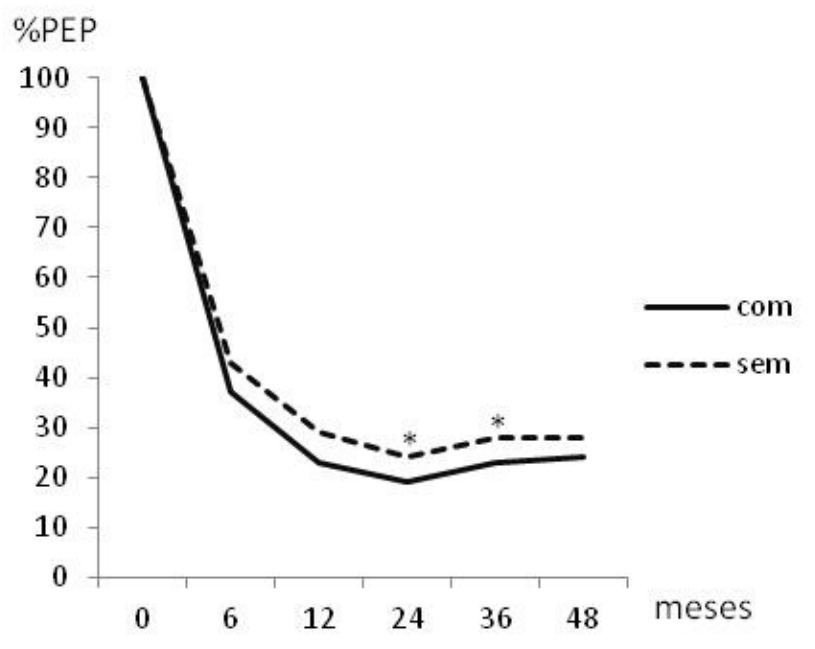

FIGURA 1 - Evolução mediana da perda do excesso de peso conforme o tipo de operação, com anel $(n=49)$ e sem anel $(n=38)$. O excesso de peso foi calculado a partir da Metropolitan Height and Weight tables, 1983. $\rho<0.05$ no teste de Friedman, sendo confirmada a diferença dos tempos 0 e $6 \mathrm{~m}$ em relação aos subsequentes. * $=\rho<0.05$ pelo teste de Mann Whitney na comparação com e sem anel

TABELA 2 - Perda do excesso de peso nos grupos sem anel e com anel

\begin{tabular}{|c|c|c|c|c|c|c|c|c|c|c|c|}
\hline & \multicolumn{5}{|c|}{ Sem anel } & \multicolumn{5}{|c|}{ Com anel } & \multirow{2}{*}{$p^{*}$} \\
\hline & & $\mathrm{n}$ & $\mathrm{P} 25$ & P50 & P75 & & $\mathrm{n}$ & P25 & P50 & P75 & \\
\hline \multirow{4}{*}{$\begin{array}{l}\text { Perda do percentual } \\
\text { do excesso de peso }\end{array}$} & $12 \mathrm{~m}$ & 68 & $55 \%$ & $72 \%$ & $82 \%$ & $12 \mathrm{~m}$ & 75 & $65 \%$ & $74 \%$ & $86 \%$ & 0,102 \\
\hline & $24 \mathrm{~m}$ & 61 & $55 \%$ & $76 \%$ & $88 \%$ & $24 \mathrm{~m}$ & 75 & $68 \%$ & $79 \%$ & $92 \%$ & 0,062 \\
\hline & $36 \mathrm{~m}$ & 56 & $51 \%$ & $72 \%$ & $82 \%$ & $36 \mathrm{~m}$ & 75 & $65 \%$ & $76 \%$ & $88 \%$ & 0,026 \\
\hline & $48 \mathrm{~m}$ & 52 & $49 \%$ & $71 \%$ & $80 \%$ & $48 \mathrm{~m}$ & 49 & $62 \%$ & $76 \%$ & $86 \%$ & 0,060 \\
\hline
\end{tabular}

*Teste de Mann-Whitney para amostras independentes. $\mathrm{m}$ = Meses após o procedimento cirúrgico 
TABELA 4 - Quantidade de excesso de peso recuperado nos grupos "com anel" e "sem anel"

\begin{tabular}{|c|c|c|c|c|c|c|c|c|c|c|c|}
\hline & \multicolumn{5}{|c|}{ Sem anel } & \multicolumn{5}{|c|}{ Com anel } & \multirow{2}{*}{$p^{*}$} \\
\hline & & $\mathrm{n}$ & $\mathrm{P} 25$ & P50 & P75 & & $\mathrm{n}$ & P25 & P50 & P75 & \\
\hline \multirow{4}{*}{$\begin{array}{c}\text { Recuperação do } \\
\text { excesso de peso } \\
\text { (em relação ao } \\
\text { menor peso) }\end{array}$} & $12 \mathrm{~m}$ & 68 & $0,0 \%$ & $0,0 \%$ & $0,0 \%$ & $12 \mathrm{~m}$ & 75 & $0,0 \%$ & $0,0 \%$ & $0,0 \%$ & 1,000 \\
\hline & $24 \mathrm{~m}$ & 61 & $0,0 \%$ & $0,0 \%$ & $0,4 \%$ & $24 \mathrm{~m}$ & 75 & $0,0 \%$ & $0,0 \%$ & $0,0 \%$ & 0,341 \\
\hline & $36 \mathrm{~m}$ & 56 & $0,0 \%$ & $3,1 \%$ & $7,9 \%$ & $36 \mathrm{~m}$ & 75 & $0,0 \%$ & $0,8 \%$ & $7,6 \%$ & 0,543 \\
\hline & $48 \mathrm{~m}$ & 52 & $0,3 \%$ & $8,4 \%$ & $11,9 \%$ & $48 \mathrm{~m}$ & 49 & $0,0 \%$ & $6,5 \%$ & $11,0 \%$ & 0,541 \\
\hline
\end{tabular}

*Teste de Mann-Whitney para amostras independentes. $\mathrm{m}$ = meses após o procedimento cirúrgico. O excesso de peso foi calculado a partir da Metropolitan Height and Weight tables, 1983. P = percentil na curva de distribuição da variável na população, onde: $\mathrm{P} 25=$ primeiro quartil $(25 \%) ; \mathrm{P} 50=$ mediana; $\mathrm{P} 75=$ terceiro quartil $(75 \%)$.

A recuperação tardia de peso teve inicio após 24 meses pós-operatório para ambos os grupos e aumenta progressivamente (tabela 4). Não houve diferença entre os grupos "com anel" e "sem anel" na quantidade de excesso de peso que foi recuperado nos diferentes tempos de análise (tabela 4). As medianas de recuperação foram de 6,5 e 8,4\% do excesso de peso, respectivamente aos grupos "com anel" e "sem anel", após 4 anos da cirurgia. Também não houve diferença entre os grupos quanto à proporção das mulheres que recuperam peso no decorrer do tempo.

Após o $4^{\circ}$ ano da DGYR, em ambos os grupos, mais de $30 \%$ das operadas tiveram recuperação de peso acima de $10 \%$ do excesso de peso. Em torno de $10 \%$ delas, a recuperação foi maior que $20 \%$ do excesso de peso que apresentavam antes da cirurgia bariátrica e que já haviam perdido até 24 meses. (tabela 5).

TABELA 5 - Frequência e proporção de peso recuperado nos grupos "com anel" e "sem anel"

\begin{tabular}{|c|c|c|c|c|c|}
\hline & \multicolumn{2}{|c|}{$\begin{array}{c}\text { Sem anel - Recuperação } \\
\text { do peso }>10 \%\end{array}$} & \multicolumn{2}{|c|}{$\begin{array}{c}\text { Com anel - Recuperação } \\
\text { do peso }>10 \%\end{array}$} & \multirow[t]{2}{*}{$p^{*}$} \\
\hline & $n$ & $\%$ & $n$ & $\%$ & \\
\hline 24 meses & 01 & 01,6 & 04 & 05,3 & 0,379 \\
\hline 36 meses & 11 & 19,6 & 12 & 16,0 & 0,341 \\
\hline \multirow[t]{2}{*}{48 meses } & 23 & 44,2 & 15 & 30,6 & 0,217 \\
\hline & \multicolumn{2}{|c|}{$\begin{array}{c}\text { Recuperação do peso } \\
>20 \%\end{array}$} & \multicolumn{2}{|c|}{$\begin{array}{c}\text { Recuperação do peso } \\
>20 \%\end{array}$} & \\
\hline 36 meses & 02 & 03,6 & 04 & 05,3 & 0,700 \\
\hline 48 meses & 05 & 09,6 & 05 & 10,2 & 1,000 \\
\hline
\end{tabular}

* Teste Exato de Fisher. Em 24 meses, apenas uma mulher do grupo sem anel recuperou mais de $20 \%$ do peso corporal

\section{DISCUSSÃO}

Os resultados deste estudo retrospectivo sugerem que a colocação do anel de contenção gástrica nas DGYR apresenta superioridade em relação à perda ponderal, porém não se diferencia da técnica sem anel em relação à estabilidade do peso perdido no período analisado. As curvas das evoluções da perda percentual do excesso de peso (Figura 1) são claras ao mostrar o posicionamento superior do grupo com anel, ainda que não estatisticamente significativas no nível de $5 \%$ em todos os tempos. Não se trata de uma conclusão definitiva sobre vantagens da utilização do anel, mas fornece indícios de que, exclusivamente para o objetivo "perda de peso", a maior restrição pode ser o caminho mais efetivo.

Bessler $^{3}$ em estudo randomizado duplo-cego com 90 superobesos submetidos à DGYR, com ou sem banda de polipropileno de $5,5 \mathrm{~cm}$, obteve resultados de percentual de perda do excesso de peso semelhantes aos 12, 24 e 36 meses deste estudo para grupos com anel e sem anel $(12 \mathrm{~m}=64 \% \times 57,7 \% ; 24 \mathrm{~m}=64,2 \times$ $57,7 \%$ e $36 \mathrm{~m}=73,3 \times 57,7)$. Da mesma maneira, a perda percentual do excesso de peso foi sempre superior para o grupo com anel, ainda que significante no nível de $5 \%$ apenas após três anos. Awad et al. ${ }^{2}$, após estudarem 244 indivíduos submetidos à DGYR, encontraram perda percentual do excesso de peso de $80,5 \%$ e $81 \%$ após 24 e 36 meses respectivamente, ambos estatisticamente significantes em comparação ao grupo sem anel, com perda percentual do excesso de peso 69,6\% e 63,9\%. Arceo-Olaiz et al. ${ }^{1}$ em estudo controlado com 60 indivíduos, não encontrou diferenças até 24 meses.

Algumas limitações deste trabalho, como o fato de ser retrospectivo, não randomizado, com amostra de conveniência e de tamanho moderado, impedem uma inferência segura destes resultados. Também se deve considerar que a perda de peso não é o único critério para medir sucesso ou resultado em cirurgia bariátrica. Entretanto os autores optaram por não incluir neste estudo o impacto sobre a evolução das comorbidezes, nem sobre a qualidade de vida ou sobre as intolerâncias alimentares em cada grupo, nem sobre as complicações relacionadas ao uso do anel, que serão objetivo de análise em futuro estudo randomizado. 0 foco do trabalho foi a verificação isolada da ação sobre a massa corporal, que per si se configura ainda como um ponto de dúvida na literatura vigente e orientar o cálculo amostral para trabalhos prospectivos.

Muito contundentes são os achados de insucessos do grupo sem anel, ao se considerar como critério de sucesso uma perda percentual do excesso de peso maior que 50\%, aceitável entre diversos investigadores ${ }^{4,15}$. Praticamente a terça parte do grupo sem anel pode ser classificada como insucesso segundo esse critério. No grupo com anel apenas 8,2\% não atingiram o valor mínimo para sucesso após 4 anos. A diferença chega a ser três vezes menor ao se comparar ao grupo sem anel, cuja técnica de DGYR é exatamente a mesma, exceto pela presença de maior restrição. Mesmo sem avaliar qualidade de vida, essa diferença na chance de se conseguir e manter uma perda do excesso de peso $>50 \%$, sugere muita relevância.

Já em relação à recuperação do peso, não houve diferença na entre os grupos sem anel e com anel. Essa recuperação tem início no segundo ano da cirurgia e progride em termos de proporção de peso recuperado (tabela 4) e prevalência (tabela 5). 
Entre as técnicas de DGYR existem variações, incluindo o comprimento e o formato do novo reservatório gástrico, além de diversos tamanhos de anastomoses gastrojejunais, com ou sem anel ou banda não ajustável de contenção. Todas estas variações podem influenciar nos resultados finais, o que nos leva à hipótese que o componente restritivo é significativamente influente. Praticamente todos os pacientes apresentam recuperação de peso após a diminuição na restrição causada pela retirada do anel por motivo de complicações ${ }^{10}$. Derivações gástricas cujos reservatórios sejam amplos, com pouca restrição, podem evoluir com perdas ponderais modestas e capacidade ingestão de alimentos sólidos elevada ${ }^{20}$, ainda que ocorra diminuição da expressão da grelina no estomago. Da mesma forma, anastomoses gastrojejunais amplas demais podem não gerar saciedade, ainda que teoricamente favoreçam uma maior liberação de Glucagon-like peptide-1 (GLP-1) pela chegada mais rápida de alimentos ao intestino delgado.

A comprovação da redução significativa e quase imediata das concentrações séricas de grelina após as DGYR ${ }^{14}$ estimulou ainda mais o questionamento sobre a real necessidade do uso de anéis de contenção, visto que o simples fato de se excluir a maior parte do fundo gástrico seria suficiente para diminuir a fome e propiciar perda ponderal. A DGYR promove condição hormonal mais favorável, menos orexigênica e mais saciogênica, oferecendo um ambiente intrínseco para a perda e manutenção do peso. Mas esse mecanismo parece ser suplantado pelo poder da restrição mecânica. Se a restrição for pequena, pode aumentar a chance de falha cirúrgica, mesmo na vigência das modificações hormonais. Se a restrição for excessiva, pode causar complicações. A DGYR, mesmo sem anel, apresenta significativo grau de restrição à ingestão de alimentos. Este trabalho retrospectivo sugere que um pouco mais de restrição, caso seja clinicamente aceitável, sem provocar complicações em taxas elevadas, pode ser benéfico para se alcançar perdas superiores a $50 \%$ do excesso de peso.

Diversos estudos mostram que a utilização do anel nas DGYR apresenta índices de complicações como estenoses, erosões e intolerâncias alimentares dentro de limites tidos como aceitáveis. Conquanto o tamanho do anel não seja consenso, o comprimento entre 6,0 a 7,0 cm está associado à menor incidência dessas complicações, mantendo sua função restritora ${ }^{8}$. A evolução até essa medida seguiu um caminho de tentativas com comprimentos menores, a partir de 5,0 $\mathrm{cm}$, em que a incidência de vômitos pode ser proibitiva. Capella e outros adotam uma banda não ajustável de polipropileno ou Marlex $₫$ no lugar do anel de Silastic $₫$, com relato de bons resultados ${ }^{3,6}$.

A recuperação do peso pode ocorrer devido a processos de adaptações fisiológicas no trato gastrointestinal que acontecem com o passar do tempo. A adoção de estilo de vida saudável fortalece o indivíduo operado contra os antigos hábitos que contribuíram para a condição de obesidade. Esse novo comportamento é fundamental para manutenção em longo prazo do peso alcançado, visto que obesidade é uma doença crônica, progressiva, que não tem cura e que necessita de tratamento especializado mesmo após a cirurgia.

Orelacionamento médico-paciente suficientemente esclarecido é um elemento importante no momento de se acordar sobre a conduta de se utilizar ou não o anel de contenção. Por hora, os autores consideram sensato sugerir que a experiência da equipe e os fatores culturais sejam considerados na hora de decidir sobre a colocação ou não da prótese, até que mais evidências científicas definam essa questão.

\section{CONCLUSÕES}

Pacientes submetidos à DGYR com o anel de contenção gástrica tiveram maior perda de peso, estatisticamente significativa apenas aos 36 meses do pós-cirúrgico, além de maior proporção de sucesso cirúrgico (perda do excesso de peso $>50 \%$ ) no período analisado. A recuperação de peso não foi diferente entre os grupos. Diante das limitações do trabalho, as conclusões devem ser interpretadas como favoráveis à utilização do anel quando se analisa exclusivamente a perda de peso, porém ainda como evidência inicial para estudos randomizados com maior número de pacientes e de variáveis de análise, incluindo-se as avaliações de qualidade de vida.

\section{REFERÊNCIAS}

1. Arceo-Olaiz R, Espana-Gomez MN, Montalvo-Hernandez J, Velazquez-Fernandez D, Pantoja JP, Herrera MF. Maximal weight loss after banded and unbanded laparoscopic Roux-en- $Y$ gastric bypass: a randomized controlled trial. Surg Obes Relat Dis. 2008;4(4):507-11.

2. Awad W, Alvaro Garay M., Cristian Martínez B., Víctor Oñate M., Iván Turu K., Julio Yarmuch G. Descenso ponderal y calidad de vida mediante la cirugía de Bypass gástrico con y sin anillo de calibración. Rev Chilena de Chirugía. 2008;60(1):17-21.

3. Bessler M, Daud A, Kim T, DiGiorgi M. Prospective randomized trial of banded versus nonbanded gastric bypass for the super obese: early results. Surg Obes Relat Dis. 2007;3(4):480-4; discussion 4-5.

4. Brolin RE, Kenler HA, Gorman RC, Cody RP. The dilemma of outcome assessment after operations for morbid obesity. Surgery. 1989;105(3):337-46.

5. Buchwald H, Williams SE. Bariatric surgery worldwide 2003. Obes Surg. 2004;14(9):1157-64.

6. Capella RF, Capella JF, Mandec H, Nath P. Vertical Banded Gastroplasty-Gastric Bypass: preliminary report. Obes Surg. 1991;1(4):389-95.

7. Christou NV, Look D, Maclean LD. Weight gain after shortand long-limb gastric bypass in patients followed for longer than 10 years. Ann Surg. 2006;244(5):734-40. 
8. Crampton NA, Izvornikov V, Stubbs RS. Silastic ring gastric bypass: a comparison of two ring sizes: a preliminary report. Obes Surg. 1997;7(6):495-9.

9. Drew RL, Linner JH. Revisional Surgery for Severe Obesity with Fascia Banded Stoma Roux-en-Y Gastric Bypass. Obes Surg. 1992;2(4):349-54.

10. Elias AAG-J, A. B.; Berti, L. V.; Oliveira, M. R.; Bertin, N. T. S.; Malheiros, C. A.; Bastouly, M. Derivações gástricas em Y- de- Roux com anel de silicone para o tratamento da obesidade: estudo das complicações relacionadas com o anel. Arquivos Brasileiros de Cirurgia Digestiva. 2011;24(4):290-5.

11. Fobi M. Why the operation I prefer is silastic ring vertical gastric bypass. Obes Surg. 1991;1(4):423-6.

12. Fobi MA. Surgical treatment of obesity: a review. J Natl Med Assoc. 2004;96(1):61-75.

13. Fobi MA. Vertical banded gastroplasty vs gastric bypass: 10 years follow-up. Obes Surg. 1993;3(2):161-4.

14. Geloneze B, Tambascia MA, Pilla VF, Geloneze SR, Repetto EM, Pareja JC. Ghrelin: a gut-brain hormone: effect of gastric bypass surgery. Obes Surg. 2003;13(1):17-22.

15. Halverson JD, Koehler RE. Gastric bypass: analysis of weight loss and factors determining success. Surgery. 1981;90(3):446-55.
16. Kaplan LM. Gastrointestinal management of the bariatric surgery patient. Gastroenterol Clin North Am. 2005;34(1):105-25.

17. Linner JR, Drew RL. Technique of anterior wall Rouxen-Y gastric bypass for the treatment of morbid obesity. Contemp Surg. 1985;26:46-59.

18. Livingston EH. Complications of bariatric surgery. Surg Clin North Am. 2005;85(4):853-68, vii.

19. Metropolitan height and weight tables. Stat Bull Metrop Life Found. 1983;64(1):3-9.

20. Muller MK, Wildi S, Scholz T, Clavien PA, Weber M. Laparoscopic pouch resizing and redo of gastro-jejunal anastomosis for pouch dilatation following gastric bypass. Obes Surg. 2005;15(8):1089-95.

21. Salinas A, Santiago $E$, Yeguez J, Antor $M$, Salinas $H$. Silastic ring vertical gastric bypass: evolution of an open surgical technique, and review of 1,588 cases. Obes Surg. 2005;15(10):1403-7.

22. Valezi AC, Mali Junior J, de Menezes MA, de Brito EM, de Souza SA. Weight loss outcome after silastic ring Rouxen-Y gastric bypass: 8 years of follow-up. Obes Surg. 2010;20(11):1491-5. 